\title{
Preoperative Lymphocyte-To-Monocyte Ratio as a Prognostic Predictor of Long-term Mortality Among Cardiac Surgery Patients: A Propensity Score Matching Analysis Based on a 4-year Follow-up Cohort
}

\section{Zhuoming Zhou}

First Affiliated Hospital of Soochow University

Mengya Liang

First Affiliated Hospital of Sun Yat-Sen University

\section{Suiqing Huang}

First Affiliated Hospital of Sun Yat-Sen University

\section{Rennan Weng}

First Affiliated Hospital of Sun Yat-Sen University

Jian Hou

First Affiliated Hospital of Sun Yat-Sen University

Zhongkai Wu ( $\square$ wuzhk@mail.sysu.edu.cn )

The First Affiliated Hospital of Sun Yat-sen University https://orcid.org/0000-0003-1184-1182

\section{Research}

Keywords: lymphocyte-to-monocyte ratio, cardiac surgery, propensity score matching, cohort study

Posted Date: October 14th, 2020

DOI: https://doi.org/10.21203/rs.3.rs-90894/v1

License: (c) (i) This work is licensed under a Creative Commons Attribution 4.0 International License. Read Full License 


\section{Abstract}

\section{Background}

Cardiac surgery is associated with a specific inflammatory response. The lymphocyte-to-monocyte ratio (LMR) has been shown to be related to the prognosis of many cardiovascular diseases. We aimed to evaluate the prognostic value of the preoperative LMR in patients who underwent cardiac surgery.

\section{Methods}

Clinical data were extracted from the Multiparameter Intelligent Monitoring in Intensive Care (MIMIC)-III database. The optimum cutoff value of the LMR for 4-year mortality was determined by X-tile software. The Cox proportional hazard model was applied for the identification of independent prognostic factors of 4-year mortality. Survival curves were estimated using the Kaplan-Meier method, and receiver operating characteristic (ROC) curves were constructed. The 1:1 propensity score matching (PSM) method was performed to balance the influence of selection bias and potential confounding factors.

Results

A total of 1701 patients were included. The X-tile software indicated that the optimum cutoff value of the LMR for 4-year mortality was 3.58. After PSM, 489 pairs of score-matched patients were generated. The Cox proportional hazard model showed that patients with an LMR $<3.58$ had a significantly higher 4-year mortality than patients with an $L M R \geq 3.58$ in the entire cohort $(H R=1.925,95 \% \mathrm{Cl}: 1.509-2.456, p<$ $0.001)$ and the PSM subset ( $\mathrm{HR}=1.568,95 \% \mathrm{Cl}: 1.2-2.05, \mathrm{p}=0.001)$. The Kaplan-Meier survival curves showed that patients with an LMR $<3.58$ had a significantly lower 4-year survival rate in the entire cohort $(71.7 \%$ vs. $88.5 \%, P<0.001)$ and the PSM subset $(73.2 \%$ vs. $81.4 \%, P=0.002)$.

\section{Conclusions}

A lower LMR (< 3.58) was associated with a higher risk of 4-year mortality and can potentially predict the long-term mortality of cardiac surgery patients.

\section{Background}

Cardiac surgery, especially with cardiopulmonary bypass (CPB), can provoke a nonspecific inflammatory response, and inflammatory cells play a vital role in the development of inflammation both preoperatively and postoperatively (1). Inflammatory cells, such as neutrophils, lymphocytes and monocytes, are considered typical inflammatory biomarkers and has been verified to have a close relationship with cardiovascular diseases. These cells not only can induce diseases but can also predict cardiovascular prognosis (2-4).

Recently, some markers composed of the ratios of white blood cell (WBC) subgroups have been newly introduced and demonstrated to be associated with the risks and outcomes of cardiovascular diseases 
and related surgeries. For example, for cardiac surgery patients, the value of the neutrophil-to-lymphocyte ratio (NLR) can serve as a predictor of early and late mortality (5) (6), postoperative acute kidney injury (7), and postoperative atrial fibrillation $(8,9)$, while the platelet-to-lymphocyte ratio (PLR) has been reported to be a predictor of prognosis in patients with aortic dissection (10).

The lymphocyte-to-monocyte ratio (LMR), a novel emerging inflammatory marker, has been shown to be independently related with the outcomes of various cardiovascular diseases, including coronary artery disease (11) and heart failure (12). The LMR has also been demonstrated to be associated with the inhospital mortality of patients with acute type A aortic dissection (13) and saphenous vein graft disease in patients who underwent coronary artery bypass grafting (CABG) (14). However, currently no researches reported the association between LMR and the long-term survival for cardiac surgery patients.

In the present study, we aim to investigate the association between the LMR and risk of mortality in patients who underwent cardiac surgery with CPB during a 4-year follow-up.

\section{Methods}

\section{Data source}

Data were obtained from the Medical Information Mart for Intensive Care III (MIMIC-III) database, which is a large, freely available database with more than 40000 patients who had critical care unit stays at the Beth Israel Deaconess Medical Center between 2001 and 2012, of which patients in the CareVue system were admitted between 2001 and 2008, with at least 4-year follow-up time (15). Our right to access the database and acquire the data were approved by the institutional review boards of the Massachusetts Institute of Technology (Cambridge, MA, USA) after one of our authors (Zhou) finished the online training for the Collaborative Institutional Training Initiative (CITI) program of the National Institutes of Health (NIH) (Record ID 35971811).

\section{Patient selection}

Of all patients in the MIMIC-3 database, we included patients as follows: 1) those who underwent onpump cardiac surgery; 2) age older than 18 years; 3 ) have full records of routine preoperative blood examinations within the first $24 \mathrm{~h}$ of admission; and 4) recorded between 2001 and 2008 with at least a 4-year follow-up. (Normal values of monocyte counts were defined as between $0.12^{\star} 10^{9} / \mathrm{L}$ and $0.8^{*} 10^{9} / \mathrm{L}$ and lymphocyte counts were defined as between $0.8 * 10^{9} / \mathrm{L}$ and $4.0 * 10^{9} / \mathrm{L}$.)

\section{Data extraction}

All data was inquired and extracted using the Structured Query Language (SQL), and pgAdmin4 for PostgreSQL was used as the administrative platform. The extracted data included: 1) demographics: age, gender, and ethnicity; 2) vital signs: heart rate (HR), systolic blood pressure (SBP), diastolic blood pressure (DBP), respiratory rate (RR), temperature and percutaneous oxygen saturation $\left(\mathrm{SpO}_{2}\right) ; 3$ ) 
comorbidities: congestive heart failure (CHF), cardiac arrhythmias, valvular disease, hypertension, chronic pulmonary disease, renal failure, liver disease, coagulopathy and diabetes; 4) laboratory events: peripheral white blood cell count, neutrophil count, monocyte count, lymphocyte count, platelet count, hemoglobin, serum sodium, serum potassium, serum creatinine and LMR (calculated by dividing the lymphocyte count by the monocyte count; and 5) SAPS II and SOFA scores. ICU length of stay, 90-day mortality and 4-year mortality were recorded in order to analyze the outcomes. Considering the proportion of missing data for each variable was less than $1.5 \%$, we omitted them directly in further analysis.

\section{Propensity score matching}

Considering the patient selection criteria can hardly be completely random, we applied the propensity score matching (PSM) method to balance the influence of selection bias and potential confounding factors. The PSM analysis was based on the logistics regression model, and the propensity score was calculated according to the following baseline characteristics: age, gender, ethnicity, CHF, cardiac arrhythmias, valvular disease, hypertension, chronic pulmonary disease, renal failure, liver disease, peripheral white blood cell count, platelet count, hemoglobin, serum sodium, serum potassium, serum creatinine and SAPS II score. Pairs of patients with LMR $<3.58$ or $\geq 3.58$ were derived using 1:1 matching with a caliper of 0.02. Eventually, a total of 978 patients were propensity score-matched and 489 pairs of score-matched patients were generated.

\section{Statistical analysis}

Continuous variables were presented as the mean \pm SD or median (interquartile range) and compared by t-test or Mann-Whitney $U$ test. Categorical data were presented as numbers with proportions and analyzed by $\chi 2$ test. After PSM, the paired t-test and Wilcoxon rank sum test for continuous data and the McNemar test for categorical data was used for comparison between the matched groups. The optimum cutoff value of the LMR for 4-year mortality was determined by X-tile (Version 3.6.1, Yale University School of medicine) software (16). Survival curves were estimated using the Kaplan-Meier method and compared by the log-rank test. Receiver operating characteristic (ROC) curves were constructed, and the area under the curve (AUC), sensitivity and specificity were calculated.

The Cox proportional hazard model was applied for the univariate and multivariate analyses to identify independent prognostic factors of 4-year mortality after cardiac surgery. To evaluate the association between the LMR and mortality, model 1 was adjusted for age, gender, CHF, cardiac arrhythmias, valvular disease, hypertension, renal failure and liver disease; model 2 was adjusted for age and gender. The results are presented as hazard ratios (HRs) and 95\% confidence intervals (Cls).

A subgroup analysis was also performed based on age, gender and comorbidities, including liver disease, renal failure, hypertension, diabetes, chronic pulmonary disease, $\mathrm{CHF}$, cardiac arrhythmias and valvular disease. All tests were two-sided, and p-values less than 0.05 were considered significant. All statistical analyses in our study were performed using STATA V.14.0, SPSS Statistics 22 (IBM, Chicago, IL) and GraphPad Prism 8. 


\section{Results}

\section{Characteristics of patients}

In total, 1701 patients who met the selection criteria were enrolled in our study. Based on the results calculated by the X-tile software, the optimal cutoff value of preoperative LMR for 4-year mortality was set as 3.58 (with a sensitivity of $71.1 \%$ and a specificity of $56.6 \%$ ) and divided all patients into two groups according to the LMR: $<3.58(n=571)$ and $\geq 3.58(n=1130)$. The baseline characteristics of enrolled patients are briefly summarized in the Table 1 , including demographics, vital signs, laboratory events, comorbidities and scores.

Significant differences in baseline characteristics could be observed between the two groups. Patients with a lower LMR tended to be older and male with a lower DBP, SpO2, lymphocyte count, hemoglobin, and serum sodium and higher NLR, PLR, WBC count, neutrophil count, monocyte count, serum potassium, serum creatinine, SAPS II score, and SOFA score, as well as a history of CHF, cardiac arrhythmias, valvular disease, chronic pulmonary disease, renal failure, liver disease, and coagulopathy, while tending not to have hypertension and diabetes.

\section{LMR as an independent prognosticator for 4-year mortality}

Compared with patients with $L M R \geq 3.58$, patients with $L M R<3.58$ were at higher risk of prolonged ICU stay (3.1 days vs. 2.2 days, $p<0.001), 90$-day mortality $(10.3 \%$ vs. $2.9 \%, p<0.001)$ and 4 -year mortality $(28.9 \%$ vs. $11.5 \%, p<0.001)$ (Table 2$)$.

A univariate Cox regression analysis was conducted to select the variables of prognostic value for 4-year mortality, and age $(p<0.001)$, gender $(p<0.001)$, CHF $(p<0.001)$, cardiac arrhythmias $(p<0.001)$, valvular disease $(p<0.001)$, hypertension $(p=0.008)$, renal failure $(p<0.001)$, and liver disease $(p<$ 0.001 ) were selected to be adjusted in the multivariate Cox regression analysis. The results of the univariate and multivariate analyses are summarized in Table 3. In the multivariate analysis, Model 1 was adjusted for age, gender, CHF, cardiac arrhythmias, valvular disease, hypertension, renal failure and liver disease, while Model 2 was adjusted for age and gender. Patients with an LMR $<3.58$ had significantly higher 4-year mortality compared to patients with an LMR $\geq 3.58$ (Model 1: HR $=1.925,95 \% \mathrm{Cl}$ : 1.509 2.456, $\mathrm{p}<0.001$; Model 2: $\mathrm{HR}=2.651,95 \% \mathrm{Cl}: 2.075-3.31, \mathrm{p}<0.001)$.

The Kaplan-Meier survival curves comparing the two groups are shown in Figure 1A. Patients with an LMR $<3.58$ had a significantly lower 4-year survival rate compared to patients with an LMR $\geq 3.58$ $(71.7 \%$ vs. $88.5 \%, p<0.001)$.

A subgroup analysis of age ( $<70$ years old; $\geq 70$ years old), gender and comorbidities was used for further comparison of the long-term prognosis between these groups, and the results are presented in Table 4. The 4-year mortality rate was higher in the group with a LMR $<3.58$ compared with the subgroups with a LMR $\geq 3.58$, except for the subgroup of patients with liver disease $(\mathrm{HR}=0.967,95 \% \mathrm{Cl}$ : $0.363-2.65, p=0.948)$. The interactions between the LMR and all subgroup factors were analyzed and 
significant interactions were observed only for CHF $(p=0.043)$. Female patients had a significantly higher risk of 4-year mortality with an LMR $<3.58(\mathrm{HR}=2.629,95 \% \mathrm{Cl}: 1.778-3.887, \mathrm{p}<0.001)$.

\section{Association between LMR and 4-year mortality in patients with normal lymphocyte and monocyte counts}

Considering a lower LMR may result from a reduced lymphocyte count or elevated monocyte count and they may affect late mortality independently as reported previously, we also analyzed the association between the LMR and 4-year mortality in patients with normal lymphocyte and monocyte counts $(n=$ 1451).

In the group with normal lymphocyte and monocyte counts, patients with an LMR $<3.58$ were still at higher risk of prolonged ICU stay (3.0 days vs. 2.2 days, $p<0.001), 90$-day mortality $(9.3 \%$ vs. $2.8 \%, p<$ $0.001)$ and 4 -year mortality $(27.9 \%$ vs. $10.6 \%, p<0.001)$ (Table 2$)$.

As shown in Table 3, the results of multivariate Cox regression analysis in patients with normal lymphocyte and monocyte counts were similar as before. Patients with an LMR $<3.58$ had significantly higher 4-year mortality compared to patients with an LMR $\geq 3.58$ (Model 1: $\mathrm{HR}=2.656,95 \% \mathrm{Cl}$ : 2.032 3.471, $\mathrm{p}<0.001$; Model 2: $\mathrm{HR}=2.052,95 \% \mathrm{Cl}: 1.553-2.712, \mathrm{p}<0.001)$. As shown in Figure 1B, The survival curves comparing the two groups showed that, in the group with normal lymphocyte and monocyte counts, patients with an LMR $<3.58$ also had a significantly lower 4-year survival rate compared to patients with an LMR $\geq 3.58$ (72.1\% vs. $89.4 \%, P<0.001)$.

\section{The prognostic significance of LMR after PSM}

Considering the imbalanced baseline characteristics between patients with an LMR $<3.58$ and an LMR $\geq$ 3.58, we performed a 1:1 ratio PSM to balance the potential confounding factors, and 489 pairs of scorematched patients were generated. The baseline characteristics of patients after PSM are shown in Table 5. The demographics, vital signs, comorbidities and most laboratory events were well-balanced between these two groups. Since the lymphocyte, neutrophil and monocyte counts directly influence the value of the LMR, we did not include them in the matched variables. After PSM, significant differences between the two groups can still be observed in ICU length of stay (3.0 days vs. 2.9 days, $p=0.003), 90$-day mortality ( $8.6 \%$ vs. $4.9 \%, p=0.027)$ and 4-year mortality ( $26.8 \%$ vs. $18.6 \%, p=0.002)$ (Table 2$)$.

The results of multivariate Cox regression analysis in patients after PSM indicated that a LMR $<3.58$ still remained an independent predictor of higher 4-year mortality (Model 1: $\mathrm{HR}=1.568,95 \% \mathrm{Cl}: 1.20-2.05, \mathrm{p}=$ 0.001; Model 2: $\mathrm{HR}=1.517,95 \% \mathrm{Cl}: 1.159-1.986, \mathrm{p}=0.002$ ) (Table 3). Additionally, the survival curves (Figure 1C) comparing the two groups showed that, after PSM, patients with an LMR $<3.58$ still had a significantly lower 4-year survival rate compared to patients with an $L M R \geq 3.58$ (73.2\% vs. $81.4 \%, P$ $=0.002)$.

\section{Prognostic predictive ability of LMR}


To assess the potential predictive role of the LMR for 4-year mortality and to evaluate the predictive model combining the LMR and other variables, ROC curve analysis was performed, and the area under the curve (AUC) was 0.660 for the LMR and 0.785 for an LMR $<3.58$, after combining with age, gender, $\mathrm{CHF}$, cardiac arrhythmias, valvular disease, hypertension, renal failure, and liver disease (Figure 2).

\section{Discussion}

Our study investigated the association between LMR and risk of death among on-pump cardiac surgery patients within a cohort study with a 4-year follow-up. A PSM analysis was performed to balance the underlying confounding factors, and the results showed that lower preoperative LMR was a reliable predictor of unfavorable 4-year mortality. To our knowledge, this is the first study to demonstrate the correlation between the preoperative LMR and long-term mortality of cardiac surgery patients.

Inflammatory cells, including WBCs and its subtypes (lymphocytes, monocytes and neutrophils), have been well validated to play an indispensable role in cardiovascular diseases. Monocytes, as representatives of the innate immune system, play a vital role in the progression of atherosclerosis, and elevated monocytes are strongly associated with the development of coronary artery disease $(2,17,18)$. Low lymphocyte counts have also been demonstrated to be an independent prognostic marker of heart failure and coronary artery disease $(19,20)$. The potential mechanisms of the prognostic value of LMR are not fully understood now. We hypothesis that the ratio of certain WBC subtypes like LMR and NLR reflect the inflammatory background of patients, as assumed by Gibson and his colleagues (21). The lymphocyte counts represent the general health state of patients rather than the direct protective role of lymphocytes for certain pathophysiological process (4) (22). Monocytes, as the precursor of macrophages, reflect the balance between inflammation and immunity (23). Preoperative monocytosis represent the relatively insufficiency of activated macrophages therefore inflammation overweight immunity and lead to bad prognosis of cardiac surgery patients. The LMR can better integrate the clinical significance of lymphocytes and monocytes and provide additional prognostic value. Further studies focus on the mechanism of the association between LMR and the prognosis are warranted in the future.

The LMR and NLR, which are novel markers defined by the ratio of WBC subtypes, have been shown in previous studies to have prognostic value with regard to cardiovascular outcomes (2-4). Silberman and his colleagues, after a 16-year follow-up, demonstrated that the NLR can predict both early and late outcomes in patients who underwent heart surgery (5). Lin et al. discovered the correlation between the LMR and in-hospital mortality of patients with acute type A aortic dissection (13). Oksuz et al. suggested that LMR levels were independent predictors of saphenous vein graft patency in patients who underwent CABG (14). Other studies also indicated that the LMR was associated with the prognosis of patients with heart failure, myocardial infarction and stable coronary artery disease $(12,24,25)$.

The lower value of LMR may result from an elevated monocyte count or lower lymphocyte count. Both elevated monocytes and low lymphocytes are associated with a bad prognosis with regard to cardiovascular events, as reported before (2-4). Therefore, in order to discover whether the LMR itself can 
provide additional information, regardless of the elevation of monocytes and reduction of lymphocytes, we set a cohort which only included patients with normal lymphocyte and monocyte counts and discovered that a lower LMR was still associated with higher mortality in this cohort.

Another characteristic of our study was that we used the PSM analysis, which can minimize confounding factors in the baseline characteristics. The major results before and after PSM were basically consistent, but there were also differences that deserved further discussion. The HRs of an LMR $<3.58$ on the 4-year mortality before and after PSM differed from each other (Model 1: 2.656 vs. 1.568; Model 2: 2.052 vs. 1.517), which may be due to not only the balance of baseline characteristics but also the variation of the best cutoff value after PSM. Actually, due to the relatively small sample size and single-center-based cohort of the current study, the cutoff value of the LMR in our study may vary according to different strategies of matching. Further studies based on larger populations with external validation are warranted.

In our study, lower preoperative LMR was proven to be associated with a higher risk of long-term mortality. However, the results of ROC curve analysis showed that the AUC was 0.660 for the LMR only, which means that a model containing only the LMR was not enough for prediction. Therefore, we added the variables in model 1 to set a more convincing model with an AUC of 0.785 . Using only the LMR, it can be difficult to precisely predict long-term mortality, but the LMR can easily be acquired from routine blood examination, making it a simple and cost-effective indicator for prognostic evaluation. Furthermore, the results of the present study indicated the LMR may serve as an additional marker with the ability to provide information on preoperative inflammation and potentially increase the predictive accuracy of traditional models and risk scores, such as the EuroSCORE II. Unfortunately, since the MIMICIII database lacked intraoperative information, we could not build a model containing all variables in the EuroSCORE II for further analysis.

As shown in the results of the subgroup analysis, the LMR maintained its predictive capacity regardless of age, gender and most comorbidities. We found that compared with patients with CHD, a lower LMR value for patients without CHD may be more associated with a bad prognosis. CHF was one of the major adverse cardiovascular events (MACE), which was regarded as an important risk factor for long-term mortality for cardiac surgery patients. However, for patients without MACE, the predictive capacity of traditional models tended to be not as significant as patients with MACE. Our results demonstrated that a lower LMR had better predictive value in patients without CHF; therefore, it is reasonable to include the LMR in the models when considering the prognosis of patients without heart failure. Additionally, although a significant difference wasn't observed in the $p$ for interaction, we noticed an interesting result -female patients had an approximately 2.6-fold higher risk of 4-year mortality with an LMR $<3.58$, while males patients only a 1.6-fold higher risk of 4-year mortality. Further research focusing on the correlation between sex and LMR are warranted in the future.

In summary, our findings demonstrated, for the first time, that a lower LMR was correlated with a higher risk of death among on-pump cardiac surgery patients within a cohort study with a 4-year follow-up. 
However, we have to admit that our study still had several limitations. First, the information on the WBC subtypes for some patients was missing or inappropriate for further analysis. Therefore, we directly deleted them in our cohorts, which may result in selection bias and this is one of the reasons why we chose the PSM analysis for balance. Second, considering the difference between test equipment and standards among various institutions, as well as our relatively small sample size and single-center-based cohort, we cannot deny the possibility that the best cutoff value may vary based on different study populations. Further studies and external validation based on multicenter large cohorts are required to determine the most appropriate cutoff value of the LMR according to different populations. Third, restricted by the contents of the MIMIC-III database, some important information, including inflammatory biomarkers, dynamic changes of the LMR, intraoperative data and postoperative complications, were recorded incompletely and not suitable for analysis.

\section{Conclusions}

In conclusion, our findings showed, for the first time, that a lower LMR $(<3.58)$ was correlated with a higher risk of death among on-pump cardiac surgery patients within a cohort study with a 4-year followup. The LMR is a potential predictive biomarker for the long-term mortality of cardiac surgery patients and may improve the predictive ability of traditional models or risk scores. Studies of large multicenter populations with longer follow-up are warranted for further validation.

\section{Abbreviations}

LMR: Iymphocyte-to-monocyte ratio; MIMIC: Multiparameter Intelligent Monitoring in Intensive Care; ROC: receiver operating characteristic; PSM: propensity score matching; CPB: cardiopulmonary bypass; WBC: white blood cell; NLR: neutrophil-to-lymphocyte ratio; PLR: platelet-to-lymphocyte ratio; CABG: coronary artery bypass grafting; CHF: congestive heart failure; AUC: area under the curve; HRs: hazard ratios; Cis: confidence intervals; MACE: major adverse cardiovascular events

\section{Declarations}

Ethic to approval and consent to participate: The establishment of the database was approved by the Massachusetts Institute of Technology (Cambridge, MA) and the Institutional Review Boards of Beth Israel Deaconess Medical Center (Boston, MA).

Consent for publication: Not applicable

Availability of data and materials: The dataset generated or analysed during the current study are available from the Multiparameter Intelligent Monitoring in Intensive Care (MIMIC)--III database (https:// mimic.physionet.org/.).

Competing interests: The authors declare that there are no competing interests 
Funding: This work was supported by National Key R\&D Program of China [grant numbers 2017YFC1105000] and the National Natural Science Foundation of China [grant numbers 81770319 , 81570039, 81900294].

Author's contribution: (I) Conception and design: ML, ZZ; (II) Administrative support: ZW; (III) Provision of study materials or patients: ML, ZW, JH; (IV) Collection and assembly of data: ZZ, SH; (V) Data analysis and interpretation: ZZ, SH, RW; (VI) Manuscript writing: All authors; (VII) Final approval of manuscript: All authors.

Acknowledgments: Not applicable

\section{References}

1. Wan S, LeClerc JL, Vincent JL. Inflammatory response to cardiopulmonary bypass: mechanisms involved and possible therapeutic strategies. Chest. 1997; 112(3):676-92.

2. Ghattas A, Griffiths HR, Devitt A, Lip GY, Shantsila E. Monocytes in coronary artery disease and atherosclerosis: where are we now? Journal of the American College of Cardiology. 2013;62(17):1541-51.

3. Guasti L, Dentali F, Castiglioni L, Maroni L, Marino F, Squizzato A, et al. Neutrophils and clinical outcomes in patients with acute coronary syndromes and/or cardiac revascularisation. A systematic review on more than 34,000 subjects. Thrombosis and haemostasis. 2011;106(4):591-9.

4. Ommen SR, Gibbons RJ, Hodge DO, Thomson SP. Usefulness of the lymphocyte concentration as a prognostic marker in coronary artery disease. The American journal of cardiology. 1997;79(6):812-4.

5. Silberman S, Abu-Yunis U, Tauber R, Shavit L, Grenader T, Fink D, et al. Neutrophil-Lymphocyte Ratio: Prognostic Impact in Heart Surgery. Early Outcomes and Late Survival. The Annals of thoracic surgery. 2018;105(2):581-6.

6. Green J, Bin Mahmood SU, Mori M, Yousef S, Mangi AA, Geirsson A. Stability across time of the neutrophil-lymphocyte and lymphocyte-neutrophil ratios and associations with outcomes in cardiac surgery patients. Journal of cardiothoracic surgery. 2019;14(1):164.

7. Engin M. Are Pre and Postoperative Platelet to Lymphocyte Ratio and Neutrophil to Lymphocyte Ratio Associated with Early Postoperative AKI Following CABG? Brazilian journal of cardiovascular surgery. 2020;35(2):239.

8. Weedle RC, Da Costa M, Veerasingam D, Soo AWS. The use of neutrophil lymphocyte ratio to predict complications post cardiac surgery. Annals of translational medicine. 2019;7(23):778.

9. Liu Z, Nguyen Khuong J, Borg Caruana C, Jackson SM, Campbell R, Ramson DM, et al. The Prognostic Value of Elevated Perioperative Neutrophil-Lymphocyte Ratio in Predicting Postoperative Atrial Fibrillation After Cardiac Surgery: A Systematic Review and Meta-Analysis. Heart, lung \& circulation. 2019. 
10. Bedel C, Selvi F. Association of Platelet to Lymphocyte and Neutrophil to Lymphocyte Ratios with InHospital Mortality in Patients with Type A Acute Aortic Dissection. Brazilian journal of cardiovascular surgery. 2020;34(6):694-8.

11. Kurtul A, Duran M. The correlation between lymphocyte/monocyte ratio and coronary collateral circulation in stable coronary artery disease patients. Biomarkers in medicine. 2017;11(1):43-52.

12. Silva N, Bettencourt P, Guimaraes JT. The lymphocyte-to-monocyte ratio: an added value for death prediction in heart failure. Nutrition, metabolism, and cardiovascular diseases : NMCD. 2015;25(11):1033-40.

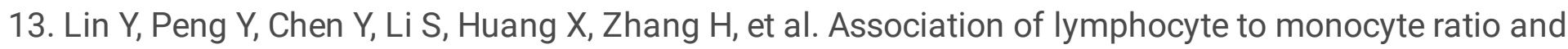
risk of in-hospital mortality in patients with acute type $A$ aortic dissection. Biomarkers in medicine. 2019;13(15):1263-72.

14. Oksuz F, Elcik D, Yarlioglues M, Duran M, Ozturk S, Celik IE, et al. The relationship between lymphocyte-to-monocyte ratio and saphenous vein graft patency in patients with coronary artery bypass graft. Biomarkers in medicine. 2017;11(10):867-76.

15. Johnson AE, Pollard TJ, Shen L, Lehman LW, Feng M, Ghassemi M, et al. MIMIC-III, a freely accessible critical care database. Scientific data. 2016;3:160035.

16. Camp RL, Dolled-Filhart M, Rimm DL. X-tile: a new bio-informatics tool for biomarker assessment and outcome-based cut-point optimization. Clinical cancer research : an official journal of the American Association for Cancer Research. 2004;10(21):7252-9.

17. van der Laan AM, Hirsch A, Robbers LF, Nijveldt R, Lommerse I, Delewi R, et al. A proinflammatory monocyte response is associated with myocardial injury and impaired functional outcome in patients with ST-segment elevation myocardial infarction: monocytes and myocardial infarction. American heart journal. 2012;163(1):57-65.e2.

18. Maekawa Y, Anzai T, Yoshikawa T, Asakura Y, Takahashi T, Ishikawa S, et al. Prognostic significance of peripheral monocytosis after reperfused acute myocardial infarction:a possible role for left ventricular remodeling. Journal of the American College of Cardiology. 2002;39(2):241-6.

19. Vaduganathan M, Ambrosy AP, Greene SJ, Mentz RJ, Subacius HP, Maggioni AP, et al. Predictive value of low relative lymphocyte count in patients hospitalized for heart failure with reduced ejection fraction: insights from the EVEREST trial. Circulation Heart failure. 2012;5(6):750-8.

20. Núñez J, Miñana G, Bodí V, Núñez E, Sanchis J, Husser O, et al. Low lymphocyte count and cardiovascular diseases. Current medicinal chemistry. 2011;18(21):3226-33.

21. Gibson PH, Croal BL, Cuthbertson BH, Small GR, Ifezulike Al, Gibson G, et al. Preoperative neutrophillymphocyte ratio and outcome from coronary artery bypass grafting. American heart journal. 2007;154(5):995-1002.

22. Coller BS. Leukocytosis and ischemic vascular disease morbidity and mortality: is it time to intervene? Arteriosclerosis, thrombosis, and vascular biology. 2005;25(4):658-70.

23. Guilliams M, Mildner A, Yona S. Developmental and Functional Heterogeneity of Monocytes. Immunity. 2018;49(4):595-613. 
24. Kose N, Akin F, Yildirim T, Ergun G, Altun I. The association between the lymphocyte-to-monocyte ratio and coronary artery disease severity in patients with stable coronary artery disease. European review for medical and pharmacological sciences. 2019;23(6):2570-5.

25. Fan Z, Li Y, Ji H, Jian X. Prognostic utility of the combination of monocyte-to-lymphocyte ratio and neutrophil-to-lymphocyte ratio in patients with NSTEMI after primary percutaneous coronary intervention: a retrospective cohort study. BMJ open. 2018;8(10):e023459.

\section{Tables}

Table 1. Baseline characteristics before propensity score matching $(n=1701)$. 


\begin{tabular}{|c|c|c|c|}
\hline \multirow[t]{2}{*}{ Characteristics } & \multicolumn{2}{|l|}{ LMR } & \multirow[t]{2}{*}{$\mathrm{p}$-value } \\
\hline & $<3.58(\mathrm{n}=571)$ & $\geq 3.58(n=1130)$ & \\
\hline \multicolumn{4}{|l|}{ Demographics } \\
\hline Age, years & $69.3(61.2-77)$ & $65.8(56.3-74.3)$ & $<0.001$ \\
\hline Male, n (\%) & 422 (73.9) & $756(66.9)$ & 0.003 \\
\hline Ethnicity, n (\%) & & & 0.008 \\
\hline White & $428(75)$ & $816(72.2)$ & \\
\hline Black & $10(1.8)$ & $54(4.8)$ & \\
\hline Others & $133(23.3)$ & $260(23)$ & \\
\hline \multicolumn{4}{|l|}{ Vital Signs } \\
\hline HR, beats/minute & $85.1(79.5-91.4)$ & $85.4(79.7-91.4)$ & 0.640 \\
\hline $\mathrm{SBP}, \mathrm{mmHg}$ & $111.7(106.1-120)$ & $111.3(105.7-119.1)$ & 0.299 \\
\hline $\mathrm{DBP}, \mathrm{mmHg}$ & $55.8(51.7-60.2)$ & $56.6(52.7-61)$ & 0.016 \\
\hline $\mathrm{RR}$, times/minute & $17(15.5-19.4)$ & $16.7(15.1-18.8)$ & 0.003 \\
\hline Temperature, ${ }^{\circ} \mathrm{C}$ & $36.9(36.6-37.2)$ & $36.9(36.6-37.2)$ & 0.321 \\
\hline $\mathrm{SpO} 2, \%$ & $98.2(97.4-99)$ & $98.4(97.5-99.1)$ & 0.048 \\
\hline \multicolumn{4}{|l|}{ Comorbidities } \\
\hline Congestive heart failure & $267(46.8)$ & $297(26.3)$ & $<0.001$ \\
\hline Cardiac arrhythmias & $343(60.1)$ & $493(43.6)$ & $<0.001$ \\
\hline Valvular disease & $169(29.6)$ & $225(19.9)$ & $<0.001$ \\
\hline Hypertension & $380(66.5)$ & $793(70.2)$ & 0.127 \\
\hline Chronic pulmonary disease & $117(20.5)$ & $164(14.5)$ & 0.002 \\
\hline Renal failure & $96(16.8)$ & $70(6.2)$ & $<0.001$ \\
\hline Liver disease & $24(4.2)$ & $30(2.7)$ & 0.850 \\
\hline Coagulopathy & $45(7.9)$ & $58(5.1)$ & 0.025 \\
\hline Diabetes & 188 (32.9) & $397(35.1)$ & 0.365 \\
\hline
\end{tabular}




\begin{tabular}{|c|c|c|c|}
\hline LMR & $2.6(2-3.1)$ & $5.4(4.5-7)$ & $<0.001$ \\
\hline NLR & $5.6(3.8-9.2)$ & $2.5(1.8-3.6)$ & $<0.001$ \\
\hline PLR & $181.7(137.5-255.1)$ & $120.5(92.4-156.7)$ & $<0.001$ \\
\hline WBC, $10^{9} / \mathrm{L}$ & $8.9(6.7-12.2)$ & $7.5(6-9.4)$ & $<0.001$ \\
\hline Neutrophils, \% & $77.2(70.1-84.1)$ & 66 (59.1-72.8) & $<0.001$ \\
\hline Monocytes, \% & $5.5(4.2-7)$ & $4.6(3.7-5.5)$ & $<0.001$ \\
\hline Lymphocytes, \% & $13.7(9-18.7)$ & $25.7(20.4-31.8)$ & $<0.001$ \\
\hline Platelets, $10^{9} / \mathrm{L}$ & $221(175-282)$ & $228(190.8-275.3)$ & 0.056 \\
\hline Hemoglobin, g/dL & $10.1(9.2-11.1)$ & $10.5(9.5-11.5)$ & $<0.001$ \\
\hline Serum sodium, $\mathrm{mmol} / \mathrm{L}$ & 137.5 (135.5-139) & $137.5(136-139)$ & 0.013 \\
\hline Serum potassium, $\mathrm{mmol} / \mathrm{L}$ & $4.6(4.2-4.9)$ & $4.5(4.2-4.8)$ & 0.033 \\
\hline Serum creatinine, $\mathrm{mg} / \mathrm{dL}$ & $1(0.8-1.4)$ & $0.9(0.7-1.1)$ & $<0.001$ \\
\hline \multicolumn{4}{|l|}{ Scores } \\
\hline SAPS II & $36(29-43)$ & $31(25-39)$ & $<0.001$ \\
\hline SOFA & $5(3-7)$ & $4(3-6)$ & $<0.001$ \\
\hline
\end{tabular}

Values are presented as the mean \pm standard deviation, median (interquartile range), or number of patients (\%).

Abbreviations: HR, heart rate; SBP, systolic blood pressure; DBP, diastolic blood pressure; RR, respiratory rate; Sp02, percutaneous oxygen saturation; LMR, lymphocyte-to-monocyte ratio; NLR, neutrophil-tolymphocyte ratio; PLR, platelet-to-lymphocyte ratio; WBC, white blood cell; SAPS II, Simplified Acute Physiology Score II; SOFA, Sequential Organ Failure Assessment.

Table 2. Outcomes of patients before and after PSM matched and patients with normal lymphocyte and monocyte counts. 


\begin{tabular}{|c|c|c|c|}
\hline & LMR $<3.58$ & LMR $\geq 3.58$ & $\mathrm{p}$-value \\
\hline \multicolumn{4}{|l|}{ Before PSM } \\
\hline & $N=571$ & $N=1130$ & \\
\hline ICU stay, days & $3.1(1.9-5.6)$ & $2.2(1.3-4)$ & $<0.001$ \\
\hline 90-day mortality, n (\%) & $59(10.3)$ & $33(2.9)$ & $<0.001$ \\
\hline 4-year mortality, n (\%) & $165(28.9)$ & $130(11.5)$ & $<0.001$ \\
\hline \multicolumn{4}{|l|}{ After PSM } \\
\hline & $N=489$ & $N=489$ & \\
\hline ICU stay, days & $3.0(1.7-5.2)$ & $2.9(1.7-5.3)$ & 0.003 \\
\hline 90-day mortality, n (\%) & $42(8.6)$ & $24(4.9)$ & 0.027 \\
\hline 4-year mortality, $\mathrm{n}(\%)$ & $131(26.8)$ & $91(18.6)$ & 0.002 \\
\hline \multicolumn{4}{|c|}{ Normal lymphocytes and monocytes group } \\
\hline & $N=393$ & $N=1058$ & \\
\hline ICU stay, days & $3.0(1.6-5.2)$ & $2.2(1.3-3.9)$ & $<0.001$ \\
\hline 90-day mortality, n (\%) & $37(9.3)$ & $29(2.8)$ & $<0.001$ \\
\hline 4-year mortality, n (\%) & $111(27.9)$ & $112(10.6)$ & $<0.001$ \\
\hline
\end{tabular}

Table 3. Univariate and multivariate Cox regression analyses for 4-year mortality in cardiac surgery patients. 


\begin{tabular}{|c|c|c|c|c|c|c|}
\hline \multirow[b]{2}{*}{ LMR $<3.58$} & \multicolumn{6}{|c|}{ Matched group $(n=978)$} \\
\hline & 0.002 & $\begin{array}{l}1.533(1.173- \\
2.003)\end{array}$ & 0.002 & $\begin{array}{l}1.517(1.159- \\
1.986)\end{array}$ & 0.001 & $\begin{array}{l}1.568(1.200- \\
2.050)\end{array}$ \\
\hline Age & $<0.001$ & $\begin{array}{l}1.043(1.029- \\
1.057)\end{array}$ & $<0.001$ & $\begin{array}{l}1.042(1.028- \\
1.056)\end{array}$ & $<0.001$ & $\begin{array}{l}1.038(1.024- \\
1.052)\end{array}$ \\
\hline Gender (male) & $<0.001$ & $\begin{array}{l}0.476(0.363- \\
0.623)\end{array}$ & 0.004 & $\begin{array}{l}0.656(0.494- \\
0.872)\end{array}$ & $<0.001$ & $\begin{array}{l}0.581(0.440- \\
0.767)\end{array}$ \\
\hline $\mathrm{CHF}$ & 0.001 & $\begin{array}{l}1.580(1.215- \\
2.056)\end{array}$ & 0.041 & $\begin{array}{l}1.323(1.012- \\
1.729)\end{array}$ & & \\
\hline Arrhythmia & 0.001 & $\begin{array}{l}1.625(1.225- \\
2.157)\end{array}$ & 0.099 & $\begin{array}{l}1.280(0.955- \\
1.715)\end{array}$ & & \\
\hline $\begin{array}{l}\text { Valvular } \\
\text { disease }\end{array}$ & $<0.001$ & $\begin{array}{l}1.690(1.283- \\
2.227)\end{array}$ & 0.005 & $\begin{array}{l}1.493(1.130- \\
1.973)\end{array}$ & & \\
\hline Hypertension & 0.040 & $\begin{array}{l}0.754(0.575- \\
0.988)\end{array}$ & 0.003 & $\begin{array}{l}0.649(0.489- \\
0.862)\end{array}$ & & \\
\hline Renal failure & $<0.001$ & $\begin{array}{l}2.190(1.582- \\
3.030)\end{array}$ & $<0.001$ & $\begin{array}{l}2.250(1.603- \\
3.158)\end{array}$ & & \\
\hline \multirow[t]{2}{*}{ Liver disease } & $<0.001$ & $\begin{array}{l}2.967(1.783- \\
4.935)\end{array}$ & $<0.001$ & $\begin{array}{l}3.444(2.038- \\
5.818)\end{array}$ & & \\
\hline & \multicolumn{6}{|c|}{ Normal lymphocytes and monocytes group $(n=1451)$} \\
\hline LMR $<3.58$ & $<0.001$ & $\begin{array}{l}2.923(2.248- \\
3.801)\end{array}$ & $<0.001$ & $\begin{array}{l}2.052(1.553- \\
2.712)\end{array}$ & $<0.001$ & $\begin{array}{l}2.656(2.032 \\
3.471)\end{array}$ \\
\hline Age & $<0.001$ & $\begin{array}{l}1.050(1.037- \\
1.064)\end{array}$ & $<0.001$ & $\begin{array}{l}1.043(1.029- \\
1.057)\end{array}$ & $<0.001$ & $\begin{array}{l}1.041(1.028- \\
1.055)\end{array}$ \\
\hline Gender (male) & 0.002 & $\begin{array}{l}0.659(0.503- \\
0.863)\end{array}$ & 0.045 & $\begin{array}{l}0.748(0.563- \\
0.993)\end{array}$ & 0.021 & $\begin{array}{l}0.718(0.543- \\
0.951)\end{array}$ \\
\hline $\mathrm{CHF}$ & $<0.001$ & $\begin{array}{l}2.551(1.962- \\
3.317)\end{array}$ & 0.003 & $\begin{array}{l}1.515(1.147- \\
2.003)\end{array}$ & & \\
\hline Arrhythmia & $<0.001$ & $\begin{array}{l}2.056(1.564- \\
2.704)\end{array}$ & 0.087 & $\begin{array}{l}1.286(0.964- \\
1.715)\end{array}$ & & \\
\hline $\begin{array}{l}\text { Valvular } \\
\text { disease }\end{array}$ & $<0.001$ & $\begin{array}{l}2.065(1.567- \\
2.722)\end{array}$ & 0.005 & $\begin{array}{l}1.500(1.130- \\
1.991)\end{array}$ & & \\
\hline Hypertension & 0.019 & $\begin{array}{l}0.720(0.548- \\
0.947)\end{array}$ & $<0.001$ & $\begin{array}{l}0.510(0.380- \\
0.684)\end{array}$ & & \\
\hline Renal failure & $<0.001$ & $\begin{array}{l}3.479(2.539- \\
4.767)\end{array}$ & $<0.001$ & $\begin{array}{l}2.562(1.814- \\
3.618)\end{array}$ & & \\
\hline Liver disease & $<0.001$ & $\begin{array}{l}3.447(2.072- \\
5.733)\end{array}$ & $<0.001$ & $\begin{array}{l}3.555(2.092- \\
6.042)\end{array}$ & & \\
\hline
\end{tabular}


Model I was adjusted for age, gender, CHF, cardiac arrhythmias, valvular disease, hypertension, renal failure and liver disease.

Model 2 was adjusted for age and gender.

Abbreviations: HR, hazard ratio; $\mathrm{Cl}$, confidence interval; LMR, lymphocyte-to-monocyte ratio; CHF, congestive heart failure.

Table 4. Subgroup analysis for the effect of LMR on 4-year mortality in cardiac surgery patients. 


\begin{tabular}{|c|c|c|c|c|c|}
\hline \multirow[t]{2}{*}{ Subgroups } & \multirow[t]{2}{*}{$\begin{array}{l}\text { No. of } \\
\text { patients }\end{array}$} & \multirow[t]{2}{*}{$\begin{array}{l}\text { Mortality } \\
\text { (\%) }\end{array}$} & \multicolumn{2}{|c|}{$\begin{array}{l}\text { Lymphocyte-to-monocyte ratio < } \\
3.58\end{array}$} & \multirow[t]{2}{*}{$\begin{array}{l}\mathrm{p} \text { for } \\
\text { interaction }\end{array}$} \\
\hline & & & HR (95\%Cls) & $\mathrm{p}$ & \\
\hline Age (years) & & & & & 0.327 \\
\hline$<70$ & 1004 & $110(11.0)$ & $1.840(1.225-2.764)$ & 0.003 & \\
\hline$\geq 70$ & 697 & $185(26.5)$ & $1.980(1.460-2.684)$ & $<0.001$ & \\
\hline Gender & & & & & 0.205 \\
\hline Female & 523 & $118(22.6)$ & $2.629(1.778-3.887)$ & $<0.001$ & \\
\hline Male & 1178 & $177(15.0)$ & $1.664(1.220-2.270)$ & 0.001 & \\
\hline Liver disease & & & & & 0.360 \\
\hline Yes & 54 & $25(46.3)$ & $0.967(0.353-2.650)$ & 0.948 & \\
\hline No & 1647 & $270(16.4)$ & $2.061(1.599-2.655)$ & $<0.001$ & \\
\hline Renal failure & & & & & 0.175 \\
\hline Yes & 166 & $65(39.2)$ & $1.611(0.922-2.814)$ & 0.094 & \\
\hline No & 1535 & $230(15.0)$ & $2.044(1.563-2.673)$ & $<0.001$ & \\
\hline Hypertensior & & & & & 0.782 \\
\hline Yes & 1173 & $185(15.8)$ & $1.838(1.346-2.511)$ & $<0.001$ & \\
\hline No & 528 & $110(20.8)$ & $2.131(1.433-3.169)$ & $<0.001$ & \\
\hline Diabetes & & & & & 0.710 \\
\hline Yes & 585 & 115 (19.7) & $1.471(1.003-2.159)$ & 0.048 & \\
\hline No & 1116 & $180(16.1)$ & $2.386(1.738-3.276)$ & $<0.001$ & \\
\hline Chronic pulm & ary disease & & & & 0.544 \\
\hline Yes & 281 & $70(24.9)$ & $1.386(0.847-2.268)$ & 0.194 & \\
\hline No & 1420 & $225(15.8)$ & $2.114(1.596-2.801)$ & $<0.001$ & \\
\hline Congestive $\mathrm{h}$ & t failure & & & & 0.043 \\
\hline Yes & 564 & $150(26.6)$ & $1.538(1.094-2.164)$ & 0.013 & \\
\hline No & 1137 & $145(12.8)$ & $2.374(1.693-3.331)$ & $<0.001$ & \\
\hline Cardiac arrhy & mia & & & & 0.420 \\
\hline Yes & 836 & $193(23.1)$ & $1.986(1.470-2.685)$ & $<0.001$ & \\
\hline No & 865 & $102(11.8)$ & $1.935(1.272-2.944)$ & 0.002 & \\
\hline
\end{tabular}




\begin{tabular}{|c|c|c|c|c|}
\hline Yes & 394 & $111(28.2)$ & $1.346(0.899-2.016)$ & 0.150 \\
\hline No & 1307 & $184(14.1)$ & $2.429(1.788-3.300)$ & $<0.001$ \\
\hline
\end{tabular}

HRs ( $95 \% \mathrm{Cls}$ ) were derived from Cox proportional hazards regression models and covariates were adjusted as in model 1 (Table 2 ).

Abbreviation: $\mathrm{HR}$, hazard ratio; $\mathrm{Cl}$, confidence interval.

Table 5. Baseline characteristics after propensity score matching $(n=978)$. 


\begin{tabular}{|c|c|c|c|}
\hline \multirow[t]{2}{*}{ Characteristics } & \multicolumn{2}{|l|}{ LMR } & \multirow[t]{2}{*}{$\mathrm{p}$-value } \\
\hline & $<3.58(n=489)$ & $\geq 3.58(n=489)$ & \\
\hline \multicolumn{4}{|l|}{ Demographics } \\
\hline Age, years & 68.7(61-76.9) & 69.7(60.9-77.6) & 0.482 \\
\hline Male, n (\%) & $356(72.8)$ & $373(76.3)$ & 0.218 \\
\hline Ethnicity, n(\%) & & & 0.051 \\
\hline White & $372(76.1)$ & $353(72.2)$ & \\
\hline Black & $8(1.6)$ & $17(3.5)$ & \\
\hline Others & $109(22.3)$ & $119(24.3)$ & \\
\hline \multicolumn{4}{|l|}{ Vital Signs } \\
\hline HR, beats/minute & $85(79.4-91.5)$ & 85.1(79.1-91.7) & 0.992 \\
\hline $\mathrm{SBP}, \mathrm{mmHg}$ & 111.7(106.3-120) & 112.1(106.2-120.1) & 0.493 \\
\hline $\mathrm{DBP}, \mathrm{mmHg}$ & $56.2(51.9-60.3)$ & $55.9(52.4-60.6)$ & 0.699 \\
\hline $\mathrm{RR}$, times/minute & 16.9(15.4-19.3) & $16.8(15-19)$ & 0.202 \\
\hline Temperature, ${ }^{\circ} \mathrm{C}$ & $36.9(36.6-37.2)$ & $36.9(36.6-37.3)$ & 0.561 \\
\hline SpO2, \% & 98.3(97.3-99) & 98.2(97.4-99) & 0.260 \\
\hline \multicolumn{4}{|l|}{ Comorbidities } \\
\hline Congestive heart failure & $201(41.1)$ & $202(41.3)$ & 0.999 \\
\hline Cardiac arrhythmias & $283(57.9)$ & $286(58.5)$ & 0.886 \\
\hline Valvular disease & $130(26.6)$ & $122(24.9)$ & 0.059 \\
\hline Hypertension & $317(64.8)$ & $335(68.5)$ & 0.247 \\
\hline Chronic pulmonary disease & $99(20.2)$ & 95(19.4) & 0.811 \\
\hline Renal failure & $59(12.1)$ & $59(12.1)$ & 0.999 \\
\hline Liver disease & $17(3.5)$ & $16(3.3)$ & 0.999 \\
\hline Coagulopathy & $33(6.7)$ & $43(8.8)$ & 0.289 \\
\hline Diabetes & 158(32.3) & 184(37.6) & 0.092 \\
\hline
\end{tabular}




\begin{tabular}{|c|c|c|c|}
\hline \multicolumn{4}{|l|}{ Laboratory Events } \\
\hline LMR & $2.7(2.1-3.1)$ & $4.5(4-5.1)$ & $<0.001$ \\
\hline NLR & $5.3(3.6-8.5)$ & $3.2(2.4-4.3)$ & $<0.001$ \\
\hline PLR & $182.2(138-256.8)$ & $128.1(93.3-169)$ & $<0.001$ \\
\hline WBC, $10^{9} / \mathrm{L}$ & $8.6(6.5-11.4)$ & $8.3(6.7-10.6)$ & 0.125 \\
\hline Neutrophils, \% & $76.3(69.2-83.4)$ & $70.1(63.9-76.2)$ & $<0.001$ \\
\hline Monocytes, \% & $5.7(4.4-7.1)$ & $4.8(3.8-5.8)$ & $<0.001$ \\
\hline Lymphocytes, \% & $14.5(9.8-19.2)$ & $21.9(17.7-26.3)$ & $<0.001$ \\
\hline Platelet, $10^{9} / \mathrm{L}$ & $221(178-280)$ & $222(189-272.5)$ & 0.757 \\
\hline Hemoglobin, g/dL & 10.2(9.3-11.2) & $10.4(9.4-11.2)$ & 0.614 \\
\hline Serum sodium, $\mathrm{mmol} / \mathrm{L}$ & 137.5(135.5-139) & $137.5(135.5-138.5)$ & 0.613 \\
\hline Serum potassium, $\mathrm{mmol} / \mathrm{L}$ & $4.5(4.2-4.9)$ & $4.5(4.3-4.9)$ & 0.871 \\
\hline Serum creatinine, $\mathrm{mg} / \mathrm{dL}$ & $1(0.8-1.3)$ & $1(0.8-1.2)$ & 0.710 \\
\hline \multicolumn{4}{|l|}{ Scores } \\
\hline SAPS II & $35(29-42)$ & $34(29-43)$ & 0.857 \\
\hline SOFA & $5(3-7)$ & $5(3-7)$ & 0.666 \\
\hline
\end{tabular}

Values are presented as the mean \pm standard deviation, median (interquartile range), or number of patients (\%).

Abbreviations: HR, heart rate; SBP, systolic blood pressure; DBP, diastolic blood pressure; RR, respiratory rate; SPO2, percutaneous oxygen saturation; LMR, lymphocyte-to-monocyte ratio; NLR, neutrophil-tolymphocyte ratio; PLR, platelet-to-lymphocyte ratio; WBC, white blood cell; SAPS II, Simplified Acute Physiology Score II; SOFA, Sequential Organ Failure Assessment.

\section{Figures}



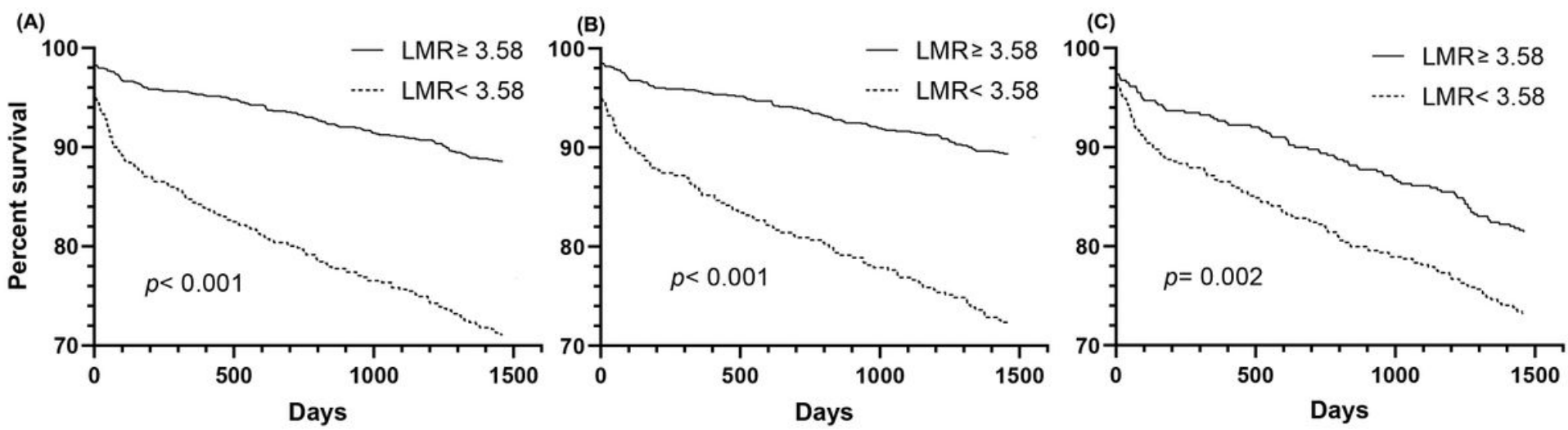

Figure 1

Kaplan-Meier survival analysis plot for 4-year overall survival. A significantly lower 4-year survival rate can be observed in the LMR $<3.58$ group compared to the $L M R \geq 3.58$ group in patients before PSM (A), patients with normal lymphocyte and monocyte counts (B) and patients after PSM (C). P-value was calculated by log-rank test and indicated in the plot. LMR, lymphocyte-to-monocyte ratio; PSM, propensity score matching 


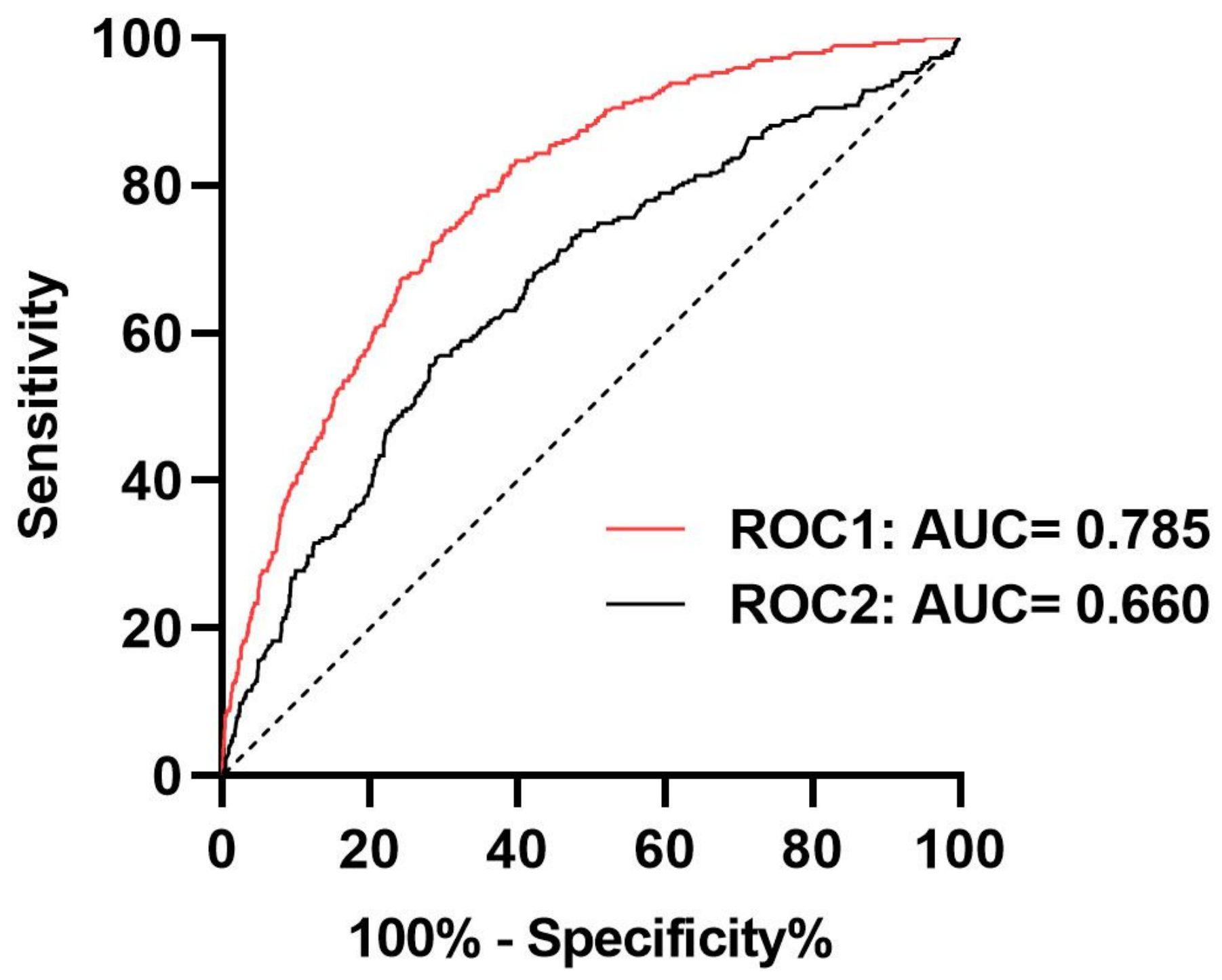

Figure 2

The receiver operating characteristic curves of predictive value of LMR for 4-year mortality of cardiac surgery patients. ROC1 included the LMR, age, gender, congestive heart failure, cardiac arrhythmias, valvular disease, hypertension, renal failure and liver disease; ROC2 included only the LMR. LMR, lymphocyte-to-monocyte ratio; AUC: area under curve; ROC: receiving operating characteristic. 